\title{
Nonlocal modeling of a Carbon Nanotube actuated by an electrostatic force
}

\author{
Hassen Ouakad $^{1, a}$, Fehmi Najar ${ }^{2}$, and Sami El-Borgi ${ }^{3,2}$ \\ 1 Mechanical Eng. Dep., King Fahd University of Petroleum and Minerals, P.O. Box 31261, Dhahran, Saudi Arabia. \\ 2 Applied Mechanics and Systems Research Lab., Tunisia Polytechnic School, University of Carthage, La Marsa, Tunisia. \\ 3 Mechanical Eng. Program, Texas A\&M University at Qatar, Eng. Building, P.O. Box 23874, Education City, Doha, Qatar.
}

\begin{abstract}
Carbon nanotubes (CNTs) are promising mechanical structures at the nano-scale which have attracted increasing attention due to their amazing mechanical, chemical, thermal, and electrical properties. To take into account size dependence of such small sized structures, the use of nonlocal continuum theory is proposed where intrinsic length scales is taken into account. Based on the Eringen theory, a nonlinear nonlocal model of a clamped-clamped CNT is developed in this study. Static and free vibration responses are simulated and analyzed. The main objective of this work is to study the influence of CNT size and length scale parameter on the static and free vibration response to better understand their effect on the general behavior of the CNT. It has been found that the nonlocal effect can largely influence the performance of the CNT and change qualitatively its nonlinear response.
\end{abstract}

\section{Introduction}

Carbon nanotubes (CNTs) are viewed as promising mechanical structures in the nano-scale which have attracted the engineers and researchers in this field mostly due to their amazing mechanical, chemical, thermal, and electrical properties. Many engineering applications have reported the possible use of CNTs as building block for novel nanoscale devices [1, 3]. Therefore, studying properly the structural behaviors of CNTs under various conditions is a fundamental problem in any nano?scale investigation. For example, many conducted experiments on CNT structures acknowledged their size dependent behavior in the nanoscale.

On the other hand, the conventional continuum mechanics, adopted and assumed in many previous investigations [4. 5], fails to predict the size dependence of such small-sized structures due to lacking of intrinsic length scales. Therefore, only recently, several higher-order elasticity theories have been presented to develop size-dependent continuum models. In what follows is a brief summary of selected studies in which nonlocal elasticity dierential model was used to model size dependence in CNTs based nanostructures. The outcomes of the below investigations are important in mechanical design considerations of devices that use CNTs as main building structures.

Numerous studies investigated the linear free and vibration and wave propagation of CNTs [6-9]. Thongyothee et al. [6] investigated the free vibration problem of CNTs including the effect of nonlocal elasticity to study the effect of their chirality and various boundary conditions. Ansari et al. [7] investigated the free vibration of double-walled carbon nanotubes (DWCNTs) using the Eringen's nonlocal elastic model along with the Donnell shell model. They accounted for the van der Waals forces between the inner and outer nanotubes. Lim and Yang [8] discussed the physics

\footnotetext{
a e-mail: houakad@kfupm.edu.sa
}

and understanding of nonlocal nanoscale wave propagation in CNTs based on nonlocal elastic stress field theory. In this regards, they developed an analytical nonlocal shear deformable nanobeam model based on the variational principle for wave propagation in CNTs. Further works focused on the nonlocal effect on the buckling characteristics of CNTs considering linear theory [9]. In a more recent work, Kiani [10] developed several novel models, all based on the nonlocal stress theory, to study the lateral buckling of groups of vertically aligned single-walled carbon nanotubes (SWCNTs). In the model, he accounted for the effect of $\mathrm{vdW}$ forces along with the nonlocal Rayleigh beam theory.

The previous concisely summarized works are all assuming linear problems. Those who included nonlinearity in either the geometry or the actuating force for CNTs are few [11-17]. To mention some, Hosseini-Ara et al. [11] proposed analytical solutions based on nonlocal Timoshenko kinematics, strain gradient approach and some variational methods to derive the higher-order boundary conditions as well as governing for the sake of investigating the buckling characteristics of CNTs. Karlicic et al. [12] analyzed the free flexural vibration and buckling SWCNT under compressive axial loading. Ansari et al. [13] studied numerically the torsional vibration behaviors of SWCNTs. They assumed an Euler?Bernoulli model while including the material length scale parameters through the strain gradient elasticity theory as to capture the CNT material size dependent effect. Fakhrabadi et al. [14] presented an investigation into the free and forced nonlinear vibration of CNTs under step electrostatic actuation based on the modified couple stress theory. Askari et al. [15] accounted for Eringen nonlocal elasticity and reformulated the classical Euler-Bernoulli beam theories to carry out the nonlinear free vibration analysis of a SWCNT. Mehdipour et al. [16] investigated the use electrostatically actuated clamped-free CNT with an attached mass as a bio-mass sensor. Ke et al. [17] investigated the nonlinear free vibration problem 
Table 1: Geometrical and physical parameters.

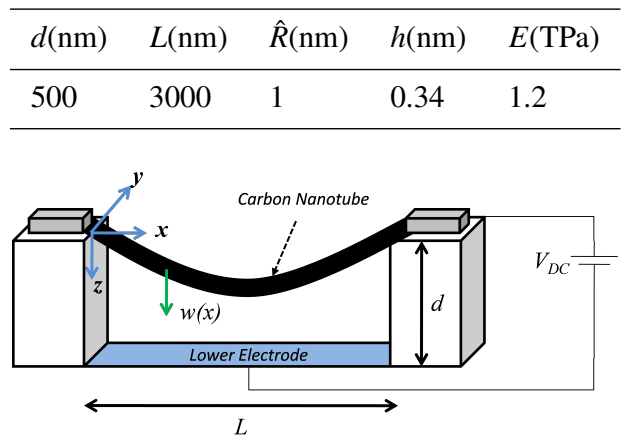

Fig. 1: 3D schematic of an electrostatically actuated straight clamped-clamped CNT actuator.

of functionally graded nanocomposite beams reinforced by SWCNTs considering the Timoshenko beam theory and von Karman geometric nonlinearity

\section{Problem Formulation}

In this section, we formulate the governing equation of an electrostatically actuated CNT actuator, shown in Fig. 1. The nanotube is triggered by an electrode underneath it with a gap width $d$. It is modeled as a hollow cylinder Euler-Bernoulli beam of radius $\hat{R}$, shell thickness $h$, and length $L$. It has a cross-sectional area $A$ and an area moment of inertia $I$. The nanotube is assumed to have a Young's modulus $E$. The CNT is considered here to be straight without slack (initial curvature). Following [18], we calculate the the nonlocal equation describing the inplane bending deflection $w(x)$ of the clamped-clamped CNT actuator. As in [18] the Eringen's theory is adopted.

$$
\begin{aligned}
\ddot{w}+w^{i v}-\mu_{0} \ddot{w}^{\prime \prime}= & V_{D C}^{2}\left(\Gamma_{e}(w)-\mu_{0} \Gamma_{e}^{\prime \prime}(w)\right) \\
& -\alpha_{1}\left(w-\mu_{0} w^{\prime \prime}\right)^{\prime \prime} \int_{0}^{1} w w^{\prime \prime} d x
\end{aligned}
$$

The boundary conditions are

$$
w(0)=0, \quad w(1)=0, \quad w^{\prime}(0)=0, \quad w^{\prime}(1)=0
$$

where the longitudinal coordinates $x$ is normalized with respect to the length $l . w(x)$ is the nondimesional transverse deflection toward the substrate and $V_{D C}$ is the applied DC voltage amplitude.

Also the function describing the variation of the electrostatic force is given by

$$
\Gamma_{e}(w)=\frac{\alpha_{2}}{\sqrt{(1-w)(1-w+2 R)}\left(\cosh ^{-1}\left(1+\frac{1-w}{R}\right)\right)^{2}}
$$

The nondimensional coefficients are defined by

$$
\alpha_{1}=\frac{A d^{2}}{2 I}, \quad \alpha_{2}=\frac{\pi \epsilon_{0} L^{4}}{E I d^{2}}, \quad R=\frac{\hat{R}}{d}, \quad \mu_{0}=\frac{\hat{\mu}_{0}}{L^{2}}
$$

where $A=\pi\left(\hat{R}+\frac{h}{2}\right)^{2}-\pi\left(\hat{R}-\frac{h}{2}\right)^{2}$ is the cross-section area, $I=\frac{\pi}{4}\left(\left(\hat{R}+\frac{h}{2}\right)^{4}-\left(\hat{R}-\frac{h}{2}\right)^{4}\right)$ is the moment of inertia, $\epsilon_{0}$ is the air permittivity and $\hat{\mu}_{0}$ is the nonlocal parameter.

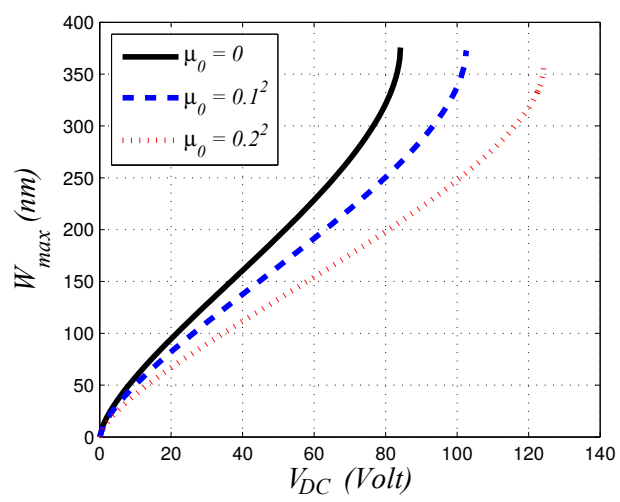

Fig. 2: Variation of the CNT maximum static deflection with the DC voltage and for different values of the nonlocal parameter.

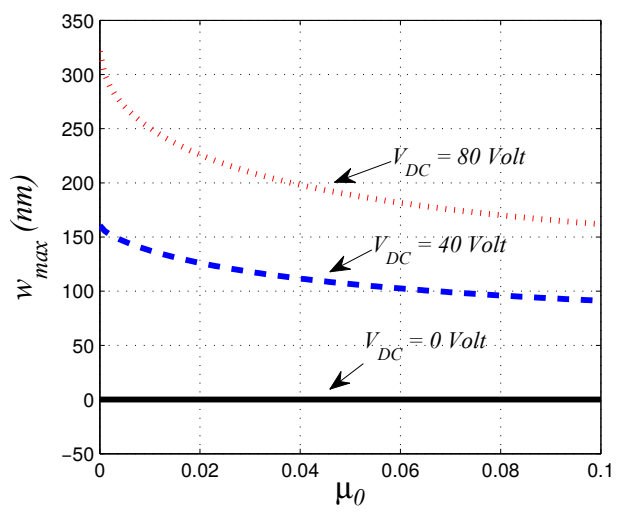

Fig. 3: Variation of the CNT maximum static deflection with the nonlocal parameter and for different values of the DC voltage.

\section{Results}

\subsection{Static Response}

We drop time dependent terms in Eq. (1) and end up with a nonlinear ODE describing the static deflection of the CNT. We use the Differential Quadrature Method (DQM) to solve for the equilibrium positions (see [18] for details of the DQM formulation). Fig. 2 shows the maximum static deflection $w_{\max }$ at midpoint as a function of the applied DC voltage and for different values of the nondimensional nonlocal parameter $\mu_{0}$. It is worth noting here that the classical theory corresponds to the case where $\mu_{0}=0$. It is clear from Fig. 2 that for a fixed DC voltage, increasing the value of the nonlocal parameter has a tendency to decrease the maximum deflection indicating that the CNT beam becomes stiffer due essentially to variation of the effective nonlinear stiffness.

Similarly in Fig. 3 illustrates the variation of the maximum static deflection $w_{\max }$ at midpoint in terms of the nondimensional nonlocal parameter $\mu_{0}$ and for different values of the applied DC voltage. It is evident that the effect of the nonlocal parameter becomes more pronounced for increasing values of the applied DC voltage. 


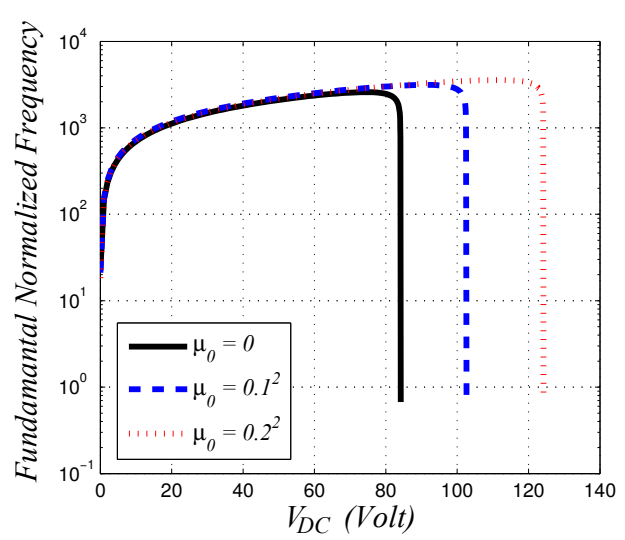

Fig. 4: Variation of the CNT fundamental normalized frequency with the DC voltage and for different values of the nonlocal parameter.

\subsection{Linear Natural Frequencies}

We start by linearizing Eq. (1) around the stable equilibrium position obtained by solving the static response of the CNT. Therefore, the associated linear eigenvalue problem is solved for the natural frequencies of the CNT using the DQM [18]. As a result, the linear nonlocal effect in both midplane stretching (cubic nonlinearity) and the actuating electric force (quadratic nonlinearity) are taken into account.

Fig. 4 depicts the variation of the fundamental normalized frequency of the CNT beam, for different values of the applied DC voltage and the nonlocal parameter. The fundamental frequency clearly increases for increasing DC voltage before the occurrence of pull-in. Increasing the values of the nonlocal parameter allows a slight increase in the frequency and a further growth of the pull-in voltage. This indicates that the effect of the nonlocal parameters has a tendency to make the CNT beam more rigid, which is the same conclusion reached in the static analysis.

For higher natural frequencies and different values of the nonlocal parameter, we show in Fig. 5 the influence of the applied DC voltage. As expected, the frequencies increase for high values of the DC voltage. Furthermore, the nonlocal parameter tends to reduce the frequencies and this effect is more significant in higher modes. To understand further this effect, the first five natural frequencies are plotted for increasing values of the nonlocal parameter in Fig. 6 and 7 for fixed values of the DC voltage of 0 and 40 volts, respectively. It is clear from the case of 0 volt DC voltage (Fig. 6) that all frequencies are reduced for increasing values of the nonlocal parameter indicating that the beam becomes more flexible which is the opposite conclusion than that of the static analysis.

We can see from Fig. 6 that without DC load, the CNT is not stretched and then the nonlocal effect will be of softening type. Whereas, if we increase the DC load to a nonzero value (see Fig. 7) the frequencies start to increase, mainly due to a hardening cubic effect of the nonlocal parameter, and then the quadratic effect will jump as the dominant for higher values of the nonlocal parameter. Adding to that the effect of the rotary inertia and because of its minus sign visible in Fig. 6, the effective stiffness of the CNT is further reduced.

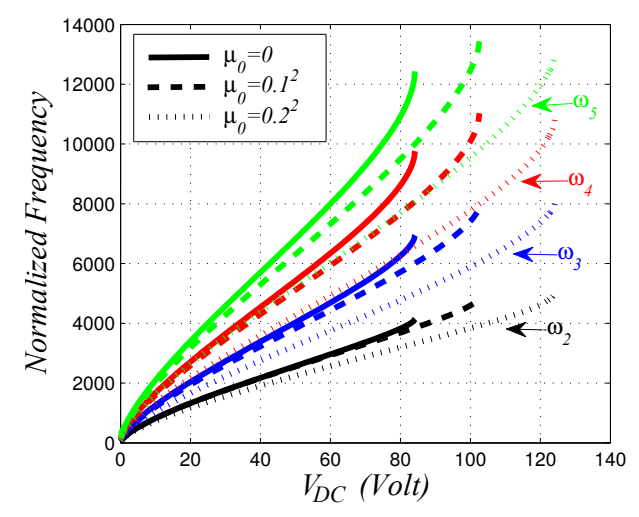

Fig. 5: Variation of the CNT normalized higher-order frequencies with the DC voltage and for different values of the nonlocal parameter.

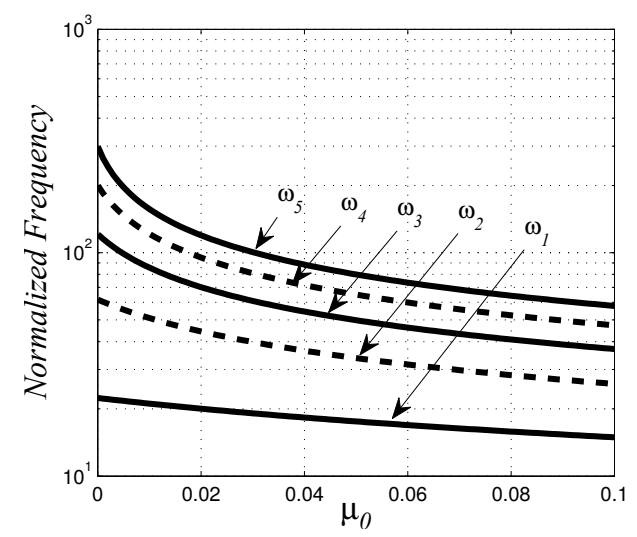

Fig. 6: Variation of the CNT normalized higher-order frequencies with the nonlocal parameter and for zero DC voltage.

For the cases of 40 volts DC voltage (Fig. 7), the same conclusion is reached except that for the fundamental frequency where an increase is first observed due to the influence of the quadratic nonlinearity of the electrostatic force. Then, when the $\mu_{0}$ is higher than 0.02 a decrease of the fundamental frequency is obtained. It is also interesting to note in Fig. 7 the cross-over of the first two natural frequencies for a value of the nonlocal parameter equal to 0.06 , the second mode of vibration becomes the fundamental mode.

Fig. 9 illustrates the effect of the gap width to CNT radius ratio on the pull-in voltage for a fixed beam length to radius ratio equal to $3000 \mathrm{~nm}$ and for different values of the nonlocal parameter. It can be observed that for small values of the gap width to CNT radius ratios, the effect of the nonlocal parameter is insignificant. However, this effect becomes significant for larger values of the ratio indicating that the pull-in voltage increases for increasing values of the nonlocal parameter.

Finally, Fig. 10 depicts the effect of the beam length to radius ratio on the pull-in voltage for a fixed the gap width to CNT radius ratio equal to $500 \mathrm{~nm}$ and for different values of the nonlocal parameter. It can be concluded that for large values of the beam length to radius ratio, the effect of the nonlocal parameter is insignificant. On the other hand, this effect becomes pronounced for small values of the ratio 


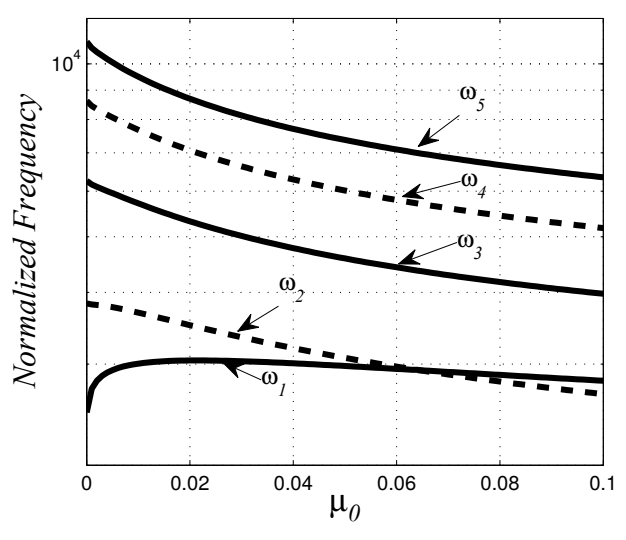

Fig. 7: Variation of the CNT normalized higher-order frequencies with the nonlocal parameter and for 80 Volt DC voltage.

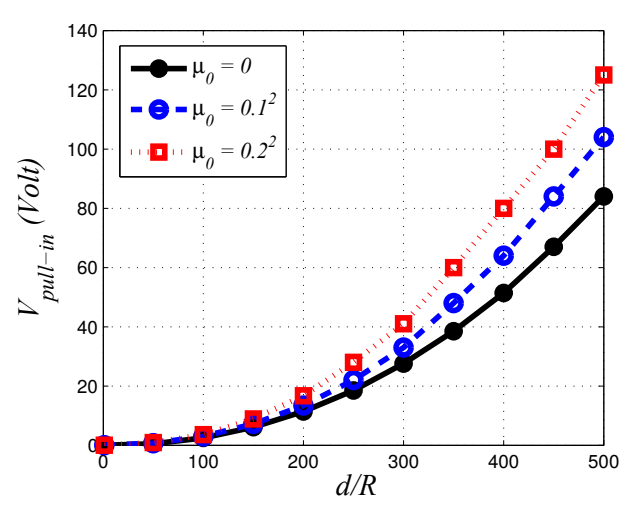

Fig. 8: Variation of the CNT pull-in voltage of the for different gap size to $\mathrm{CNT}$ radius ratio and for $\mathrm{L}=3000 \mathrm{~nm}$.

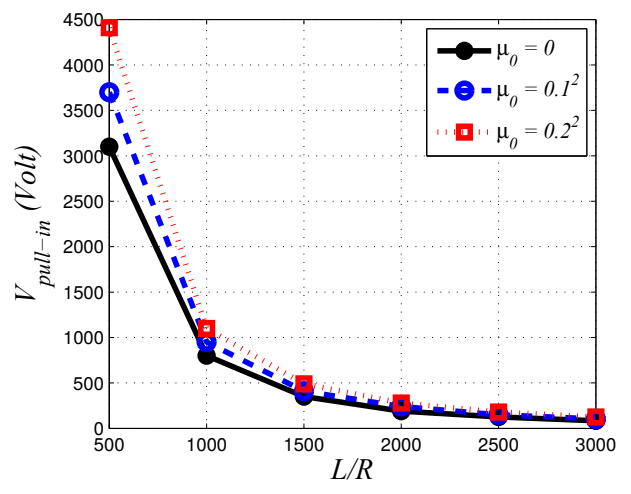

Fig. 9: Variation of the CNT pull-in voltage of the for different CNT length to radius ratio and for $\mathrm{d}=500 \mathrm{~nm}$.

indicating that the pull-in voltage increases for increasing values of the nonlocal parameter.

\section{Conclusions}

Carbon nanotubes (CNT) are mechanical structures depicting interesting behavior at the nano-scale. However, size dependence of such small sized structures can influence their responses to external excitations. The nonlocal continuum theory has been used to take into account intrinsic length scales of the CNT. Based on the Eringen theory, a nonlinear nonlocal model of a clamped-clamped CNT was developed. We used the Differential Quadrature Method to solve the derived Static and free vibration equations.

The influence of CNT size and length scale parameter on the static response has been analyzed. It has been shown that an increase of the nonlocal parameter increases the effective forcing on the CNT which implicate an apparent stiffness reduction reducing the static response and enlarging the pull-in voltage.

For free vibration responses linearized eigenvalue problem around the static solution has been solved to calculate the natural frequencies of the CNT, it has been found that the nonlocal effect reduces all natural frequencies up to the fifth mode when no external DC voltage is applied. If the linear par $t$ of the electrostatic force is taken into account, the fundamental frequency is first increased under the hardening behavior of the quadratic nonlinearity of the electrostatic force, then it start to decrease again following the general trend of the other frequencies.

\section{References}

1. V. Sazonova, Y. Yaish, H. Üstünel, D. Roundy, T. A. Arias, and P. L. McEuen, Nature 431, (2004) 284-287.

2. H. Üstünel, D. Roundy, and T. A. Arias, Nano Letters 5, (2005) 523-526.

3. Zhao, C., Song, Y., Ren, J., Qu, X., Biomaterials 30, (2009) 1739-1745.

4. H. M. Ouakad, M. I. Younis, J. Computational and Nonlinear Dynamics 5, (2010) 011009.

5. W. G. Conley, A. Raman, C. M. Krousgrill, S. Mohammadi, Nano Letters 8, (2008) 1590-1595.

6. C. Thongyothee, S. Chucheepsakul, T. Li, In Advanced Materials Research 747, (2013) 257-260.

7. Ansari, R., H. Rouhi, and B. Arash. IJST 37, 2 (2013) 91-105.

8. Lim, C. W., and Y. Yang. J. Mechanics of Materials and Structures 5, 3 (2010) 459-476. $\mathrm{y}$

9. Xu, Xiao-jian, Zi-chen Deng. Applied Mathematics and Mechanics 35, (2014) 1115-1128.

10. Kiani, Keivan. Acta Mechanica 225, 12 (2014) 35693589.

11. Hosseini-Ara, R., H. R. Mirdamadi, H. Khademyzadeh, R. Mostolizadeh. J. of the Brazilian Society of Mechanical Sciences and Engineering 34, 2 (2012) 126134.

12. D. Karlicic, P. Kozic, R. Pavlovic, J. Theoretical and Applied Mechanics 53, (2015) 217-233.

13. R. Ansari, R. Gholami, S. Ajori. J. Vibration and Acoustics 135, 5 (2013) 051016.

14. M. M. S. Fakhrabadi, A. Rastgoo, M. T. Ahmadian, and M. M. Mashhadi, Acta Mechanica 225, 6 (2014) $1523-1535$.

15. H. Askari, D. Zhang, E. Esmailzadeh, In Nanotechnology (IEEE-NANO), (2013) 545-548.

16. I. Mehdipour, A. Erfani-Moghadam, C. Mehdipour. Current Applied Physics 13, 7 (2013) 1463-1469.

17. L-L Ke, J. Yang, S. Kitipornchai, Composite Structures 92, 3 (2010) 676-683.

18. F. Najar, S. El-Borgi, JN Reddy, K. Mrabet, Composite Structures 120, (2015) 117-128. 